\title{
La genèse des neurosciences
}

\section{Entre technosciences* et diplomatie de l'innovation ${ }^{* *}$, des années 1940 aux années 1970}

> À partir des années 1940, plusieurs dynamiques permirent de renforcer le champ des sciences du cerveau et du système nerveux dans un processus interdisciplinaire, favorisé par l'idée que les biochimistes viendraient désormais éclairer les mécanismes physiologiques du système nerveux. Plus globalement, des grands programmes de recherche comme le projet Manhattan ${ }^{\star \star \star}$ ou bien la réalisation de I'ENIAC (electronic numerical integrator and computer), avaient mis en évidence l'interpénétration, sans dépendance unilatérale de l'une à l'égard de l'autre, des sciences et des techniques. L'illusion d'une technique «appliquant» les découvertes scientifiques s'évanouit alors. Le concept de «technosciences ${ }^{\star}$, en permettant de sortir de cette dichotomie, permet de mieux comprendre comment, entre les années 1940 et 1970, diverses trajectoires convergèrent pour donner naissance aux «neurosciences modernes » $[1,2]$. «

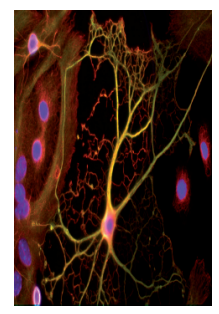

${ }^{1}$ Professeur à Sorbonne Université, président du Comité pour l'histoire de l'Inserm, Inserm, 101 rue de Tolbiac 75654 Paris Cedex 13, France.

${ }^{2}$ Secrétaire scientifique du Comité pour l'histoire de l'Inserm, Inserm, 101 rue de Tolbiac 75654 Paris Cedex 13, France.

${ }^{3}$ Professeur émérite à Sorbonne Université, membre de l'Académie des Sciences, membre fondateur de l'Institut du cerveau et de la moelle épinière, ICM, CHU Pitié-Salpêtrière, 47 boulevard de l'Hôpital, 75013 Paris, France. pascalgriset@icloud.com celine.paillette@ext.inserm.fr yves.agid@icm-institute.org
Pendant cette période, les découvertes sur les fondements physiologiques des grandes fonctions du système nerveux et, plus particulièrement du cerveau, ont été foisonnantes. La recherche française reflète à cet égard l'extraordinaire diversité des approches et des interac-

\footnotetext{
Vignette (Photo (c) Inserm/Saoudi, Yasmina).

* Le concept de technosciences, utilisé dès les années 1940, s'est largement répandu à la fin des années 1980. II souligne l'intrication entre sciences et techniques. Attribué à plusieurs auteurs, la paternité de ce terme est largement partagée. Voir [1] à propos de cette généalogie. Il a été souvent utilisé de façon critique pour questionner le contrôle de plus en plus problématique des évolutions des sciences et des techniques.

** La diplomatie de l'innovation («Innovation diplomacy»), un concept introduit au début des années 2010, qualifie une politique publique de soutien et d'incitations financière par un pays ou un groupe de pays, ici en matière de neurosciences, pour assurer à ce pays ou à ces pays, une reconnaissance et une attractivité intellectuelle et économique internationales. On pourra se référer à l'article de Jos Leijten (EurJ Fut Res, $2017 ; 5: 20$ ). La diplomatie de l'innovation a fait notamment I'objet d'un projet, l'EL-CSID (European Leadership in Cultural, Science and Innovation Diplomacy) dans le cadre du programme européen Horizon 2020.

*** Projet de recherche nucléaire débuté en 1939 par les États-Unis, avec la participation du Royaume-Uni et du Canada, qui aboutit notamment à la fabrication de la première bombe atomique.
}

tions entre les différentes disciplines contribuant à la construction des neurosciences.

Plus que leur développement, dont quelques éléments saillants, mais forcément partiels, seront mentionnés, c'est bien leur structuration en tant que champ globalement connu sous le terme de «neurosciences» qui est l'objet de cet article. Plus spécifiquement encore, il s'agira de comprendre comment cette mutation s'opéra par la politique de recherche des institutions scientifiques américaines, dans une dynamique principalement centrée sur les États-Unis ${ }^{1}$, mais avec la construction d'un réseau international, qui favorisa un large renouvellement de la science et des thérapeutiques à partir des années 1960.

\footnotetext{
Aux États-Unis, le nombre de scientifiques s'enrichit alors de tous les réfugiés en provenance de l'Europe en guerre.
} 


\section{Biologie moléculaire, pharmacologie et grands programmes, de la Seconde Guerre mondiale aux années 1960}

Les années 1950 furent marquées par le croisement de nombreuses disciplines autour d'objectifs nouveaux. Cette approche, qui avait été favorisée par les grands programmes militaires dès la Seconde Guerre mondiale, marque profondément les trajectoires scientifiques. Sciences de la vie, électronique, sciences humaines..., les hybridations deviennent courantes et font émerger des communautés de recherche qui contribuent, directement ou indirectement, à la connaissance du cerveau.

\section{L'élan de l'Entre-deux-guerres prolongé}

Au tournant des années 1940-1950, plusieurs courants disciplinaires vinrent apporter une lumière nouvelle sur le fonctionnement du cerveau et sur le comportement humain. La démonstration de l'importance de la nature chimique de la neurotransmission en constitue l'un des axes principaux. Le croisement et l'amélioration des techniques issues de l'électrophysiologie et de la biochimie permirent d'établir que la plus grande part des synapses utilise un médiateur nerveux, c'est-à-dire une transmission neuro-hormonale ([3], p. 24l). Le neurophysiologiste australien John Eccles (1903-1997), qui avait étudié sous la direction de Sherrington (1857-1952) à Oxford et avait été longtemps le fervent défenseur de la théorie électrique, se consacrait désormais aux mécanismes chimiques de la neurotransmission [4]. Les recherches sur les neuromédiateurs, dopamine, GABA, acides aminés, se développèrent. Il devenait possible de tracer les circuits cérébraux formés par des neurones produisant tel ou tel neurotransmetteurs ([3], p. 241). À partir de 1946, Julius Axelrod (1912-2004) étudie les effets secondaires des analgésiques [5], puis met en lumière le fonctionnement de neurotransmetteurs, les catécholamines. Ses travaux furent à la base du développement d'inhibiteurs sélectifs de la recapture de la sérotonine [6], les premiers antidépresseurs. Les travaux innovants se multiplièrent ainsi dès les années 1950, s'appuyant notamment sur l'expérimentation animale.

Ces approches étaient articulées à des préoccupations médicales. Aux États-Unis, le National Mental Health Act de 1946 ouvrit la voie aux études sur la santé mentale, par la fondation du National Institute of Mental Health. Les échanges interdisciplinaires se renforcèrent. En France, la neurobiochimie prenait son essor. La synthèse de la chlorpromazine par Simone Courvoisier et Paul Charpentier, chercheurs chez Rhône-Poulenc [7], I'utilisation clinique de cette molécule sur les patients atteints de psychose aiguë en 1952, effectuée à Sainte-Anne par Jean Delay (1907-1987), puis sa mise sur le marché - sous le nom de Largactil $^{\circledR}$ en France, de Thorazine ${ }^{\circledR}$ aux États-Unis - signa l'entrée dans l'ère des neuroleptiques ${ }^{2}$ [8]. La psychopharmacologie nourrit un élan optimiste chez les psychiatres, proposant une nouvelle voie thérapeu-

2 Parallèlement, Henri-Marie Laborit (1914-1995), a joué un rôle important dans le développement de la chlorpromazine, associant avec succès la prométhazine (Phénergan ${ }^{\circledR}$ ), la dibenzothiazine (Diparcol ${ }^{\circledR}$ ) et la péthidine (Dolosal ${ }^{\circledR}$ ), afin d'obtenir une hibernation artificielle pendant et après les opérations chirurgicales; médecin, il s'est rendu célèbre par ses recherches sur le système végétatif, sa découverte de l'anesthésie potentialisée et, donc, ses travaux sur l'hibernation artificielle [44, 45]. tique, un élan contrebalancé par l'émergence du mouvement antipsychiatrique dans les années $1960^{3}$ [9].

Pour les patients atteints de la maladie de Parkinson, l'espoir d'une révolution thérapeutique s'opéra à la fin des années 1960, avec une amélioration des symptômes souvent spectaculaire des personnes traitées par l'utilisation en routine de la L-DOPA, puis de la dopamine synthétique [10]. À la fin des années 1960, furent découvertes par Roger Guillemin (né en 1924) et Andrew Schally (né en 1926) [11, 12] les endorphines, les opiacés naturels du cerveau [13], découverte pour laquelle ils reçurent en 1977 le prix Nobel. D'autres domaines progressaient également. En France, l'École de neurochirurgie de SainteAnne développait avec succès la stéréotaxie humaine, technique de repérage de l'espace cérébral. Reposant sur un appareillage complexe (cadre enserrant la tête du sujet et microélectrodes), cette technique permettait d'intervenir sur des zones très précises du cerveau. Elle est fondamentale en termes de neurologie fonctionnelle, mais aussi en termes d'exploration. En 1957, Jean Talairach (1911-2007) ${ }^{4}$, publiait avec son équipe le premier Atlas d'anatomie stéréotaxique, qui devint une référence et fut suivi de plusieurs opus $[14,15]$.

\section{De nouvelles approches de la connaissance}

En 1945, Vannevar Bush (1890-1974), directeur du US Office of Science Research and Development, en proposant le programme Science: The Endless Frontier, donna un nouvel élan à l'effort scientifique américain ${ }^{5}$. La recherche se liait dès lors de manière croissante, directement ou indirectement, au complexe militaro-industriel. Avec la construction des premiers ordinateurs pendant la Seconde Guerre mondiale est apparue l'idée que l'intelligence n'était plus l'apanage du cerveau humain [16]. Alors que la possibilité d'un cerveau artificiel semblait émerger, de multiples questions étaient formulées: l'ordinateur du futur devra-t-il copier le cerveau humain? D'autres architectures sont-elles possibles? Quelle interface, ou connexion, entre le cerveau humain et la machine? Le cognitivisme se développa dans ce contexte. Les conférences Macy [47] $(\rightarrow)$ mirent en évidence, entre 1946 et 1953,

$(\rightarrow)$ Voir le Repère de J. Haiech, $m / s$ $n^{\circ} 10$, octobre 2020 , page 919

\footnotetext{
${ }^{3}$ Ce courant de pensée, complexe et disparate, prend son essor à partir des années 1960. II conteste la psychiatrie en tant que discipline scientifique et s'oppose à ses pratiques. Ce mouvement valorise par exemple l'individu dans sa singularité, en introduisant la subjectivité dans la définition du normal et du pathologique, en considérant la folie comme construction sociale.

${ }^{4}$ Jean Talairach deviendra le directeur de l'unité de recherche Inserm « Neurophysiopathologie et thérapeutiques des épilepsies ».

${ }^{5}$ Le rapport de Vannevar Bush au président Roosevelt est devenu une référence incontournable, en faveur du développement des sciences, pour servir des intérêts géostratégiques et, plus globalement, dans l'intérêt public général. En libre accès, https://www.nsf.gov/od/lpa/nsf50/vbush1945.htm
} 
l'intérêt des approches transdisciplinaires pour bâtir une nouvelle science du fonctionnement de l'esprit humain, les travaux d'Allen Newell (1927-1992) et Herbert Simon (1916-2001)6 marquant une avancée majeure pour une application concrète du cognitivisme et une rupture assumée sur le plan opérationnel avec le behaviorisme ${ }^{7}$ [17]. Dix ans plus tôt, les théories du mathématicien Norbert Wiener (1894-1964) avaient contribué à la fondation et à la diffusion de la cybernétique, suscitant réflexions et recherches théoriques sur les fonctionnements du cerveau et des machines, ainsi que sur leurs analogies [18]. Celles-ci se firent en étroite interaction avec les neurophysiologistes et psychologues du Massachusetts Institute of Technology (MIT), dont Arturo Rosenblueth (1900-1970) et Warren McCulloch (1898-1969). Ces recherches s'inscrivirent également dans le cadre des relations privilégiées existant entre certains mathématiciens et physiologistes pressentant des analogies entre les mécanismes neuroniques et les opérations élémentaires des machines à calculer. En France, des travaux et des parcours intellectuels et scientifiques mettent en lumière la nouveauté, la complexité et les tensions suscitées par ce rapprochement entre cerveau et machine. Dès les années 1940, le mathématicien et cybernéticien Louis Couffignal (1902-1966) et le neurophysiologiste Louis Lapicque (1866-1952) multipliaient les échanges. Alfred Fessard (1900-1982), ancien élève de Lapicque et pionnier de la neurophysiologie moderne, manifesta un intérêt pour la cybernétique, invitant ainsi Wiener et McCulloch à l'Institut Marey [19]. Ces exemples démontrent aussi l'originalité et la force de démarches qui, en interaction avec la science américaine, n’en étaient nullement dépendantes et surent mettre en cause les forces trompeuses de l'analogie entre l'électronique et le physiologique, pouvant déboucher sur un inquiétant déterminisme. Georges Canguilhem (1904-1995), philosophe et docteur en médecine, s'opposera ainsi radicalement au « mécanisme » [20].

Les recherches de Douglas Engelbart (1925-2013) mirent cependant en lumière les conséquences indirectes mais très concrètes de ce foisonnement où se mêlaient intuition, sciences et conviction. Revendiquant le fait de travailler sur l'augmentation de l'intelligence humaine, celui-ci explora les pistes les plus diverses, permettant à l'utilisateur d'agir en sympathie avec la machine. Le 9 décembre 1968, il présente publiquement, lors d'un séminaire depuis dénommé The Mother of All $D e m o^{8}$, un ensemble de logiciels commandés par une souris, élément central de son «Augmented Knowledge Workshop » [21]. Plus globalement, les sciences cognitives se développaient, s'organisaient et rivalisaient pour proposer des axes conceptuellement innovants afin de mieux comprendre ce qu'est la pensée et de réinterroger son rapport au biologique [22].

\footnotetext{
${ }^{6}$ Leur article Elements of Human problem solving, Psychological Review, 65, 3, 1958 est considéré comme fondateur.

${ }^{7}$ Le behaviorisme se développe depuis le début du $x x^{e}$ siècle avec les travaux fondateurs de John Broadus Watson (1878-1958), psychologue américain. Dit simplement, le behaviorisme s'intéresse au comportement, à la relation entre un stimulus et une réponse comportementale. II donne ainsi à la psychologie un objet directement observable (contrairement à la conscience).

8 Littéralement, «la mère de toutes les démos » est le nom donné à la démonstration de Douglas Engelbart, qui s'est déroulée à San Francisco.
}

De la nouvelle biologie des années 1950 au « Mind Brain Group 》

La découverte de la structure physique en double hélice de I'ADN par Rosalind Franklin (1920-1958), James Watson (1928-) et Francis Crick (1916-2004) en 1953 marqua, de manière globale, une rupture conceptuelle radicale. II est significatif qu'elle ne commence à trouver ses effets les plus opérationnels qu'en s'appuyant sur les techniques numériques. Ce lien avec la technique s'affirmait dès les années 1950, tant la biologie fut marquée par l'utilisation du microscope électronique et plus globalement par son inclusion dans un environnement de recherche, marqué par une intégration plus poussée entre sciences et innovation technique. Nommé directeur du département de biologie du MIT au début des années 1940, Francis 0. Schmitt (1903-1995) est I'un des acteurs majeurs de ce mouvement. Alors qu'il avait été l'un des pionniers de la microscopie électronique, il est recruté au MIT, à la demande notamment de Vannevar Bush, pour engager les recherches en biologie sur des pistes radicalement nouvelles [23, 24]. L'interdisciplinarité sera au cœur de sa démarche.

«Schmitt pensait de manière globale comme certains philosophes et scientifiques des années 1930 l'avaient fait. Il s'inscrivait dans le mouvement d'Unité de la science' dont les membres plaidaient pour le rassemblement de toutes les sciences par le biais de théories générales logiques et pour l'élimination des distinctions arbitraires, même si elles étaient commodes » ([25], p. 16). Cette pensée s'articulait à une volonté concrète, qui s'enracinait dans les initiatives prises pendant la guerre par l'administration américaine pour «brasser » les équipes des différentes institutions et des différentes disciplines.

Ce fut dans cette perspective qu'il créa dans les années 1950 le Mind-Brain Group en réunissant au MIT une trentaine de scientifiques, de différents centres de recherche et de différentes nationalités. II s'agissait de réunir des scientifiques très talentueux et motivés (indépendamment de leur appartenance à une discipline officielle) et de leur poser des questions sur le cerveau et l'esprit en les laissant s'informer mutuellement. II était dès lors plus que probable que des hypothèses réalisables, de nouvelles théories et même une réelle compréhension des mécanismes sous-jacents en résulteraient. Prenons par exemple la question suivante: "Quels sont les mécanismes cérébraux impliqués dans la mémoire? Si vous réunissez un neuroanatomiste de haut niveau (Walle Nauta), un spécialiste des membranes cellulaires (Albert Lehninger), un physicien des réactions rapides (Manfred Eigen) et d'autres scientifiques remarquables, comme un physiologiste (Ross 
Adey), un zoologiste (Theodore Bullock), un neurochimiste (Seymour Kety), un psychologue (Robert Galambos), etc., vous obtiendrez peutêtre des résultats qui pourraient éclairer l'apprentissage et la mémoire à court et à long terme, tels qu'ils sont transmis par divers mécanismes du système nerveux » ([25], p. 17).

\section{De multiples initiatives à travers le monde}

La détermination et la réussite de Schmitt ne doit pas éclipser les nombreuses initiatives qui virent les chercheurs intéressés par le cerveau et la pensée se regrouper dans les pays les plus avancés au sein de clubs, d'organisations plus ou moins formelles ou d'instituts académiques ([26], p. 6-8). La recherche américaine fut la plus dynamique et attira largement les meilleurs spécialistes étrangers. Plusieurs centres se créèrent ainsi au cours des années 1950 dans un mouvement de coopération internationale. En 1953, par exemple, Louis Flexner (1902-1996) à I'université de Pennsylvanie, fondait The Institute for Neurological Sciences. Aujourd'hui connue sous le nom de Mahoney Institute for Neurosciences, cette institution attira précocement de nombreux chercheurs européens. L'exemple des travaux menés conjointement par Horace Magoun (1907-1991) et Giuseppe Moruzzi (1910-1986) sur le sommeil souligne cette capacité des États-Unis à attirer les talents. Ils amenèrent Horace Magoun à créer en 1959 le Brain Research institute à I'université de Californie à Los Angeles (UCLA).

Dans le contexte de la Guerre froide la dimension internationale de ces partenariats apparaît remarquable. Elle fut facilitée par les initiatives de la Macy Foundation de New York qui soutenait des cycles de conférences portant sur la cybernétique, la conscience, la neuropharmacologie et qui permettait d'inviter des chercheurs de tous les pays et notamment soviétiques dans les centres de recherche nord-américains. Ces rencontres trouvent leur écho en URSS où le physiologiste Ivane Beritashvili (aussi appelé Ivan Beritov) (1884-1974) organisa les conférences de Gagra sur les bords de la mer Noire, ou bien en France avec les «Colloques de Marseille» lancés par Henri Gastaut (1915-1995), à partir de 1950, qui eurent rapidement un grand rayonnement international. Le «Colloque de Moscou» de 1958 sur l'électro-encéphalogramme ( $દ € G)$ résultait de ces différentes initiatives, réunissant plus d'une quarantaine de chercheurs d'URSS, d'Europe de l'Est, d'Amérique du Nord, d'Europe occidentale, mais aussi de Chine ${ }^{9}$ et d'Inde. À cette occasion, Herbert Jasper (1906-1999), Alfred Fessard, Henri Gastaut, ainsi qu'Ivane Beritashvili ${ }^{10}$, soutinrent la création d'une organisation mondiale et interdisciplinaire pour promouvoir la recherche sur le cerveau et le comportement [27, 28].

L'International Brain Research Organization (IBRO) ${ }^{11}$ fit aboutir ces idées en 1960. L'IBRO naissait sous les auspices de l'Organisation des Nations unies pour l'éducation, la science et la culture (Unesco, pour United Nations Educational, Scientific and Cultural Organization), en 1960, avec le soutien de l'Organisation mondiale de la santé (OMS). L'appui de I'US National Institute of Mental Health et de plusieurs

\footnotetext{
9 Il s'agit de la République populaire de Chine (RPC).

${ }^{10}$ https://www.fens.org/wp-content/uploads/2020/11/Beritashvili-Ivane.pdf

11 https://ibro.org/
}

fondations témoignait de l'engagement important des États-Unis dans ce processus. L'un des premiers projets de l'IBRO fut de lancer une vaste enquête afin de recenser à travers le monde les différentes équipes, laboratoires, instituts et d'identifier leurs besoins [28].

\section{«We are neuroscientists ${ }^{12} 》$. Institutionnalisation américaine et internationalisation de la communauté neuroscientifique}

Du bouillonnement des années 1950 résulte une période de structuration et d'affirmation à partir des années 1960. Les institutions américaines y prennent une place considérable.

Le programme américain de recherche Neurosciences (Neurosciences Research Program)

Le lancement en 1962 du Neurosciences Research Program (NRP) au sein du MIT par Francis 0. Schmitt, se fit sur la base de l'expérience du Mind Brain Group qu'il institutionnalisait. C'est à ce programme qu'est souvent associée la naissance du terme «neurosciences ».

Le NRP bénéficia de financements pluriannuels du MIT, du National Institute of General Medical Sciences et d'agences telles que la NASA ([24], p. 214-216). Le NRP s'installa en dehors du campus du MIT, dans la banlieue de Boston, dans la Brandegee House, où l'Académie américaine des arts et des sciences avait ses quartiers. C'est là qu'il y tenait des séminaires, développait une bibliothèque spécialisée, se dotait peu à peu d'un personnel permanent. Et, plusieurs fois par an, le NRP organisait des sessions de travail de plusieurs jours, sur des sujets très larges ou, au contraire, très spécifiques, par exemple sur les membranes des cellules neuronales, les cellules gliales, le traitement et le stockage de l'information, les concepts mathématiques et le système nerveux central, ou l'apprentissage. Ces travaux, rencontres et échanges, étaient en partie diffusés par l'intermédiaire du NRP Bulletin. Plus qu'un institut, Francis 0 . Schmitt voyait dans le NRP « un centre d'investigation» ([29], p. V). Celui-ci marqua l'évolution du champ par son engagement constant pendant vingt ans, avec une large interdisciplinarité.

En 1967, The Neurosciences: A Study Program annonçait l'avènement du «day of the brain » ([25], p. 20). Il est couramment retenu comme le premier ouvrage majeur portant dans son titre le terme «neurosciences 》- le pluriel était alors aussi utilisé en anglais. Cet ouvrage fondateur est le fruit du premier programme d'étude

\footnotetext{
12 «Nous sommes des neuroscientifiques».
} 
intensive - quatre semaines de lectures, débats, d'échanges entre 153 chercheurs seniors et juniors, sur le campus de l'université du Colorado, planifié par le personnel et les membres du NRP, pour définir l'aire des neurosciences. Les scientifiques réunis venaient d'horizons professionnels multiples (psychiatrie, psychopharmacologie, neurologie, neuroanatomie, neurochimie, biologie cellulaire, mathématiques, etc.), mais partageaient un intérêt commun pour l'étude du cerveau, de l'esprit et du comportement. Parmi eux : John Eccles (1903-1997), alors en poste à l'Institute for Biomedical Research de Chicago sur la physiologie des synapses, George Edelman (1929-2014) en immunologie de Rockefeller University à New York, Michel Jouvet (1925-2017), directeur de l'Unité 52 de l'Inserm «Neurophysiologie expérimentale et clinique », sur le sommeil paradoxal, y participaient. L'ouvrage, fortement marqué par les questionnements liés à la biologie moléculaire, reçut une reconnaissance internationale et les opus qui suivirent furent connus comme les «Big Books of Neurosciences » [25].

\section{Les neurosciences expression d'une « Science Diplomacy $»^{13}$ américaine}

Les institutions américaines jouèrent donc un rôle majeur dans ce processus de structuration internationale du champ scientifique en « aidant» I'IBRO à développer ses activités. Francis 0. Schmitt s'y engagea directement. Il soutint la diffusion de l'information, par le biais de publications et de correspondances, en mobilisant ses relations personnelles par des voyages et des meetings à travers le monde. II retrouva par exemple Alfred Fessard et quelques figures éminentes européennes de différentes spécialités dans un colloque en 1964, à Munich ([24], p. 241). Toujours aux États-Unis, la National Academy of Sciences - National Research Council (NAS-NRC) forma le Committee on Brain Sciences chargé de mener les enquêtes lancées par l'IBRO. Ce Comité fut à l'origine de la fondation en 1969, de la Society for Neurosciences. Cette société comptait parmi ses missions le développement d'un champ interdisciplinaire large et ouvert, et la promotion d'une recherche indépendante sur le système nerveux et le comportement. Elle avait aussi une mission d'ouverture auprès d'un public plus large, en favorisant l'éducation aux neurosciences, en diffusant auprès du public des résultats et en expliquant les implications des recherches en cours. Louise Marshall (1908-2003), qui travaillait au sein de NAS-NRC, puis auprès d'Horace Magoun, joua un rôle important dans l'administration scientifique tant au sein de I'IBRO que de la Society for Neurosciences [26]. En 1976, I'IBRO fondait Neuroscience, une revue gérée par les scientifiques pour les scientifiques, dans une perspective résolument internationale, et à un prix accessible, disséminant les résultats des recherches émanant de toutes les branches des neurosciences ([30], p. 1]).

En un peu plus d'une dizaine d'années, les neurosciences s'étaient donc affirmées comme une communauté scientifique portée par un réseau international intégrant dans un dispositif souple une approche très interdisciplinaire centrée sur le système nerveux. Sans exclure d'autres champs, elle rassemblait principalement les disciplines relevant de la

${ }_{13}$ Voir le projet H2020 : https://www.insscide.eu recherche biomédicale. Clairement internationale, elle voyait s'affirmer un leadership américain.

La recherche française, une voie originale connectée à la dynamique américaine

La « centralité » des États-Unis n'étouffe pas, bien au contraire, les dynamiques européennes. Pour ne retenir que l'exemple de quelques scientifiques français, des recherches fondatrices y trouvaient une place spécifique et originale.

La force des jeunes neurosciences reposait ainsi sur la puissante émulation du «voyage en Amérique ». Parmi tant d'autres, on peut citer l'exemple de Jacques Glowinski $(1936-2020)$ [48] $(\rightarrow)$.

Ce dernier rejoignit en 1964 le $\rightarrow$ Voir le Repère de H. Chneiweiss, $m / s$ $n^{\circ} 2$, février 2021, page 185 rod ${ }^{14}$ aux $\mathrm{NIH}$, où il découvrit «la neuropharmacologie moderne, mais aussi une ambiance de recherche exceptionnelle » ([31], p. 18). Au sein du laboratoire d'Axelrod, il développa une technique d'introduction dans le cerveau de noradrénaline radio-marquée, permettant de suivre son transport et d'étudier sa localisation [32]. De retour en France en 1966, doté d'un large financement des $\mathrm{NIH}$, il constitua une équipe de recherche au Collège de France, développa des relations fructueuses avec la société Rhône-Poulenc. En 1972, son laboratoire devenait l'unité Inserm 114 [31]. De même, Jean-Pierre Changeux (né en 1936), après avoir obtenu son doctorat d'État de sciences naturelles en 1964 sous la direction de Jacques Monod (1910-1976) à l'Institut Pasteur, partit deux années aux États-Unis ${ }^{15}$, menant des travaux sur la reconnaissance et la transmission de signaux par la «synapse chimique ». De retour en France, c'est à I'Institut Pasteur, en 1970, qu'il réalise avec son équipe les travaux sur le récepteur de l'acétylcholine [33]. Autre exemple, celui de Claude Kordon (1934-2008), neuroendocrinologue de formation, qui partit pour la Californie, chez Charles Sawyer où il est l'un des premiers à développer les dosages radio-immunologiques. De retour, il créait I'unité de recherche Inserm 159 de neuroendocrinologie à I'hôpital Sainte-Anne avec Jean-Charles Schwartz (1936-), où il développa des travaux sur le rôle et la fonction des hormones, dans le système nerveux central et sur le comportement, ainsi que des études pionnières sur les interactions

\footnotetext{
${ }_{14}$ Julius Axelrod reçoit en 1970 le Prix Nobel de physiologie ou médecine pour ses travaux sur les neurotransmetteurs.

15 D'abord comme post-doctorant à l'université de Berkeley, dans le laboratoire de J. Gerhart et H. Schachman, puis comme «Assistant Professor » à l'université Columbia dans le laboratoire de David Nachmansohn, qui étudiait les composants biochimiques de la synapse de l'organe électrique de poissons, torpille et gymnote
} [46]. 
entre système immunitaire et système nerveux [34]. Ces liens transatlantiques n'impliquent pas une uniformisation des approches sur la base d'un moule américain. Michel Le Moal (né en 1934), médecin neuropsychiatre qui fit son post-doc au California Institute of Technology (le «Caltech») à Pasadena, développa ainsi des recherches croisant les données sociales aux éléments physiologiques à la base du développement d'une neurobiologie intégrative [35]. Le lien avec l'hôpital, dans une proximité constante entre fondamentalistes et praticiens, favorisait indéniablement cette diversité. Jean Talairach avec Pierre Buser (1921-2013) et Jean Bancaud (1921-1993)16 poursuivaient leurs travaux, en créant à Sainte Anne, en 1970, l'unité 97 de I'Inserm, consacrée à l'exploration stéréotaxique et les thérapeutiques chirurgicales de l'épilepsie. Dans les locaux, la «passerelle» entre l'unité de recherche et le service de neurologie marquait l'importance de l'association entre cliniciens et fondamentalistes ([14], p. 74-8). Par ailleurs, les perspectives offertes par les nouveaux dispositifs d'imagerie médicale inaugurée pendant les années 1970, tomographie axiale assistée par calculateur (TAC), imagerie par résonance magnétique nucléaire, ouvraient alors de nouveaux horizons [36]. La recherche en neurosciences liait de la sorte de manière de plus en plus étroite ses pratiques et d'une certaine manière son destin, au développement de techniques qui seront de plus en plus numérisées ([37], p. 45). Une nouvelle époque pouvait s'ouvrir.

\section{Conclusion}

La structuration des «neurosciences» entre la fin de la Seconde Guerre mondiale et les années 1970 croise donc de manière internationale, la prolongation et l'approfondissement des initiatives de l'Entre-deux-guerres, la diversité d'initiatives liées aux pratiques thérapeutiques, avec la force de proposition et de structuration venue des États-Unis. Les chercheurs travaillant avec Francis 0. Schmitt en étaient sans doute conscients. Après avoir envisagé le terme latin de «mensa», puis celui de «neurobiology», ils s'accordèrent sur le mot «neurosciences». Ils avaient de la sorte construit un outil de communication qui fit office de support, facilitant le ralliement de la communauté scientifique internationale à une convergence sous l'égide américaine. On peut y voir la mise en œuvre très pionnière d'une « diplomatie scientifique » [38], qui par la convergence des sciences, des techniques et des influences de pays ou de groupes de pays dessinait déjà les contours d'une véritable «Innovation diplomacy » [39]. Celle-ci articule deux logiques indissociables: la construction d'un champ scientifique par ses acteurs les plus dynamiques, qui permet à une nouvelle vision de la science de se diffuser et de se structurer d'une part; l'affirmation d'un modèle américain capable d'attirer les talents dépassant largement la discipline scientifique concernée et d'accroître, à de multiples niveaux, l'influence des États-Unis d'autre part. Si ce dernier point n'était vraisemblablement pas l'ob-

\footnotetext{
16 Jean Bancaud et Jean Talairach développèrent les méthodes de chirurgie du cerveau permettant d'observer dans ses dynamiques spatio-temporelles une crise d'épilepsie, en relation avec les signes cliniques. Voir sur la stéréotaxie, notre article précédent $\left(\mathrm{m} / \mathrm{s} \mathrm{n}^{\circ} 8-9\right.$, août-septembre 2021, pages 793-798) «Aux origines des neurosciences : l'héritage et les fondations, du xIx siècle aux années 1940.
}

jectif consciemment désiré par les chercheurs, il n'en constituait pas moins un résultat évidemment crucial du processus. Au-delà d'un champ scientifique ainsi affirmé, c'était en effet bien un modèle d'organisation et de financement d'une science travaillant en anglais, modèle validé par un système d'évaluation, via le système d'édition, centré outre-Atlantique, qui s'imposait. La communication de John Z. Young (1907-1997), lors du symposium organisé en 1973 au MIT autour du 70 anniversaire de Francis 0 . Schmitt, reflète toute l'efficacité de cette stratégie d'influence. Le chercheur britannique explique ainsi à quel point Schmitt incarnait à ses yeux le père fédérateur - bien plus que fondateur - d'une nouvelle communauté scientifique: «In the past, we have not been sure whether we are anatomists, physiologists or biochemists, psychologists or pharmacologists. But now, we know our identity. Largely thanks to Frank Schmitt's initiative, we are Neuroscientists » ([40], p. 40).

Cette «stabilisation » fut de courte durée. Confortées dans leur démarche par le développement de l'imagerie les «neuroscientists» allaient en effet confirmer leur force et l'impact global de la discipline. Symétriquement, les neurosciences allaient susciter des réticences, des oppositions, et se trouver confrontées à d'autres «interdisciplinarités ». Si la dimension philosophique des interrogations, sans cesse renouvelée par les avancées des neurosciences, constitue une continuité dans le temps long de l'histoire de la discipline [41], une nouvelle époque s'ouvre cependant avec les années 1980. Elle voit les neurosciences convoquées dans des débats de société, mobilisant des parties prenantes appartenant à des sphères de plus en plus diverses et parfois éloignées des milieux scientifiques ${ }^{17}$. L'Homme neuronal, publié par Jean-Pierre Changeux en 1983 [42], offre à cet égard un véritable repère. «Pour les chercheurs, écrira Marcel Henaff (19422018), l'ouvrage faisait figure de manifeste, le genre de manifeste dont la nouvelle neuroscience avait besoin pour se donner de l'ampleur et de la dynamique. Ce qui change la donne, c'est la possibilité de penser le vivant lui-même en termes strictement biochimiques. Avec les neurosciences, s'est produite à l'égard du concept d'esprit (mind) une révolution comparable à celle que les sciences linguistiques avaient opérée à l'égard du concept de langage » [43]. $\diamond$

The genesis of neurosciences: Between technosciences and innovation diplomacy, from the 1940 s to the 1970 s

\footnotetext{
17 Voir Du Cerveau aux Neurosciences : itinéraires dans la longue durée. Les Cahiers du Comité pour l'histoire de l'Inserm, 2021, 2, vol l et 2, en ligne: https://www. ipubli.inserm.fr/handle/10608/10030
} 


\section{LIENS D'INTÉRÊT}

Les auteurs déclarent n'avoir aucun lien d'intérêt concernant les données publiées dans cet article.

\section{RéFÉRENCES}

1. Raynaud D. Note historique sur le mot «technoscience ». Avril 2015, https://zilsel.hypotheses. org/1875 Consulté le 10/01/2021.

2. Shepherd GM. Creating modern neuroscience: the revolutionary 1950s. Oxford: Oxford University Press, $1994: 201$.

3. Oliverio A. La cellule de la pensée : le neurone. In : Pietro Corsi (ed). La fabrique de la pensée. La découverte du cerveau, de l'art de la mémoire aux neurosciences. Milan : Electra, $1990:$ 240-60.

4. Barbara. JG Neurotransmetteurs : la volte-face du professeur Eccles. Cerveau Psycho $2019 ; 110$. https://www.cerveauetpsycho.fr/sr/grandes-experiences-de-Zneurosciences/ neurotransmetteurs-la-volte-face-du-professeur-eccles-16797.php

5. Brodie BB, Axelrod J. The fate of acetanilide in the body. Proc Am Fed Clin Res $1947 ; 3: 58$

6. Axelrod J. Noradrenaline: fate and control of its biosynthesis. Nobel lecture 1970. https://www. nobelprize.org/uploads/2018/06/axelrod-lecture.pdf

7. Charpentier $P$, Gailliot $P$, Jacob R, et al. Recherches sur les diméthylaminopropyl-N phénothiazines substituées. CR Acad Sci (Paris) $1952 ; 235: 59-60$.

8. Poirier J, Clarac F, Barbara JG, Broussolle $\varepsilon$. Figures and Institutions of the neurological sciences in Paris from 1800 to 1950. Part IV: Psychiatry and psychology. Rev Neurol 2012 ; 168 : 389-402.

9. Goldstein J. Psychiatry. In : Bynum WF, Porter R (eds) Companion encyclopedia of the history of medicine. London : Routledge, 1993 : 1350-72.

10. Agid Y. Un exemple de succès thérapeutique : la L-DOPA. Le Courrier du CNRS $1984 ; n^{\circ} 55-56$ : 63-65. Site Histoire. Inserm.fr

11. Burgus R, Dunn TF, Desiderio D, et al. Characterization of ovine hypothalamic hypophysiotropic TSH-releasing factor. Nature $1970 ; 226: 321-5$.

12. Schally AV, Sawano S, Arimura A, et al. Isolation of growth hormone-releasing hormone (GRH) from porcine hypothalami. Endocrinology $1969 ; 84: 1493-506$.

13. Harrington A. The brain and the behavorial sciences. In : Bowler PJ, Pickstone JV (eds). The Cambridge history of science, vol. 6. The modern biological and earth sciences. Cambridge : Cambridge University Press, 2009 : 504-23.

14. Talairach J. Souvenirs des études stéréotaxiques du cerveau humain. Une vie, une équipe, une méthodologie. L'école de Sainte Anne. Paris : John Libbey Eurotext, 2007. Site Histoire. Inserm.fr.

15. Barbara JG. Localiser les enregistrements et les stimulations électriques du cerveau par la stéréotaxie. Bull Histoire Épistémologie Sciences Vie 2018 ; 2 : 169-86.

16. Campbell-Kelly M, Aspray W, Ensmenger N, Yost JR. Computer: a history of the information machine, $3^{\text {rd }}$ ed. Philadelphia : Westview Press, 2014

17. Newell A, Shaw JC, Simon HA. Elements of a theory of human problem solving. Psychol Rev 1958 ; $65: 151-66$.

18. Wiener RN. Cybernetic: or control and communication in the animal and the machine. New YorkCambridge-Paris : Wiley-Technology-MIT-Hermann, 1948.

19. Barbara JG. Alfred Fessard : regard critique sur la cybernétique et la théorie des systèmes. In Claude Debru C, Barbara JG, Chérici C (eds). Essor des neurosciences en France dans le contexte international (1945-1975). Paris : Hermann, 2008 : 135-47.

20. Canguilhem G. La connaissance de la vie. Paris : Vrin, 1952

21. Engelbart D, Englisg W. A research center for augmenting human intellect. Proceedings Fall JCC $1968: 33-1: 395-410$

22. Chamak B. Dynamique d'un mouvement scientifique et intellectuel aux contours flous : les sciences cognitives (États-Unis, France). Rev Histoire Sciences Humaines 2011 ; 2(25) : 13-33.

23. Abi-Rached R, Rose N. Historiciser les neurosciences. In : Moutaud B, Chamak B (eds). Neurosciences et société : enjeux des savoirs et pratiques sur le cerveau. Paris : Armand Colin, $2014: 51-77$

24. Schmitt F0. The never-ceasing search. Philadelphia : American Philosophical Society, 1990.

25. Adelman, G. The neurosciences research program at MIT and the beginning of the modern field of neuroscience. J History Neurosci $2010 ; 19$ : 15-23.

26. The Creation of Neuroscience. The Society for Neuroscience and the Quest for Disciplinary Unity 1969-1995, p.6-8. https://www.sfn.org/about/history-of-sfn/the-creation-of-neuroscience/ / media /SfN/Images/History\%20of\%20SfN/pdf/HistoryofSfN.ashx.
27. Chérici C. Études électrocliniques. Développements de l'électroencéphalographie et de l'épileptologie à Marseille après la Seconde Guerre mondiale. In : Claude Debru C, Barbara JG, Chérici C (eds). Essor des neurosciences en France dans le contexte international (1945-1975), Paris Hermann, 2008 : 199-211.

28. Marshall LH. Historical report. Early history of IBRO: the birth of organized neuroscience. I. The antecedent ground swell. Neuroscience 1996 ; $72: 283-6$.

29. Schmitt FO. Forword. In: Quarton GC, Melnechuk T, Schmitt FO (eds). The neurosciences: a study program. New York : Rockefeller UP, 1967.

30. Kostyuk PG, Llinás R, Smith AD. Editorial. Neuroscience ; 1976 ; 1.

31. Glowinski J. Les commencements de la neuropharmacologie. In : Debru C, Barbara JB, Chérici C (eds). L'essor des neurosciences, France, 1945-1975, Paris: Hermann, 2008 : 16-8. Site Histoire. Inserm.fr.

32. Iversen LL, Glowinski J, Axelrod J. Reduced uptake of tritiated noradrenaline in tissues of immunosympathectomized animals. Nature $1965 ; 206: 1222-3$.

33. Benda P, Tsuji S, Daussant J, Changeux JP. Localization of acetylcholinesterase by immunofluorescence in eel electroplax. Nature $1970 ; 225: 1149-50$

34. Kordon C. Site Histoire. https://histoire.inserm.fr/les-femmes-et-leshommes/claude-kordon/

35. Le Moal M. Entre le comportement et la molécule : les neurosciences intégratives peuvent-elles exister? In : Xavier Seron (ed). Psychologie et cerveau. Psychologie d'aujourd'hui. Paris: PUF, 1999 : 195-221.

36. Syrota A. Voir le fonctionnement du cerveau vivant chez l'homme : la tomographie d'émission de positons. In : Corsi P (ed). La fabrique de la pensée. La découverte du cerveau de l'art de la mémoire aux neurosciences. Milan : Electa, 1990

37. Chamak B. Neurosciences et sciences cognitives : quelle histoire et quelles orientations? In : Moutaud B, Chamak B (eds). Neurosciences et société : enjeux des savoirs et pratiques sur le cerveau. Paris : Armand Colin, 2014 $23-50$

38. Ruffini PB. Science et diplomatie : une nouvelle dimension des relations internationales. Paris : Éditions du Cygne, 2015

39. Griset P. Innovation diplomacy: a new concept for ancient practices? Hague J Diplomacy, 2020 ; 15 : 383-97.

40. Young JZ. Sources of discovery in neuroscience. In : Worden FG, Swazey JP, Adelman $\mathrm{G}$ (eds). The neurosciences: paths of discovery. Cambridge (Mass) : MIT Press, 1975

41. Debru C. Neurophilosophie du rêve. Paris : Hermann, 1990.

42. Changeux JP. L'homme neuronal. Paris : Fayard, 1983.

43. Hénaff M. Le moment du cerveau. Compte rendu de l'ouvrage : Michel Morange, Francis Wolff, Frédéric Worms (dir.). L'Homme neuronal, trente ans après. Dialogue avec Jean-Pierre Changeux. Paris : Éditions de la rue d'Ulm, 2016. Esprit 2016, ; $426: 210-3$.

44. Laborit $H$, Huguenard $P$, Alluaume $R$. Un nouveau stabilisateur végétatif (le 4560 RP). Presse Med $1952 ; 60$ : 206-8.

45. Laborit H, Huguenard P. Artificial hibernation by pharmacodynamical and physical means. Presse Med 1951 ; $59: 1329$.

46. Changeux JP. Responses of acetylcholinesterase from Torpedo marmorata to salts and curarizing drugs. Mol Pharmacol $1966 ; 2$ : 369-92.

47. Haiech J. Parcourir l'histoire de l'intelligence artificielle, pour mieux la définir et la comprendre. Med Sci (Paris) 2020 ; $36: 919-23$.

48. Chneiweiss H. Sur les épaules des géants qui nous ont précédés. Jacques Glowinski, de la neuropharmacologie biochimique à l'architecture. Med Sci (Paris) $2021 ; 37: 185-8$

\section{TIRÉS À PART}

P. Griset, C. Paillette, y. Agid

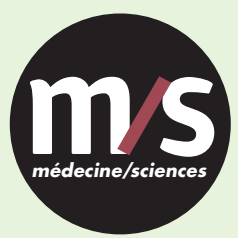

Tarifs d'abonnement $m / s-2021$

$>$ Grâce à $m / s$, vivez en direct les progrès des sciences biologiques et médicales

Abonnez-vous

à médecine/sciences

Abonnez-vous sur www.medecinesciences.org
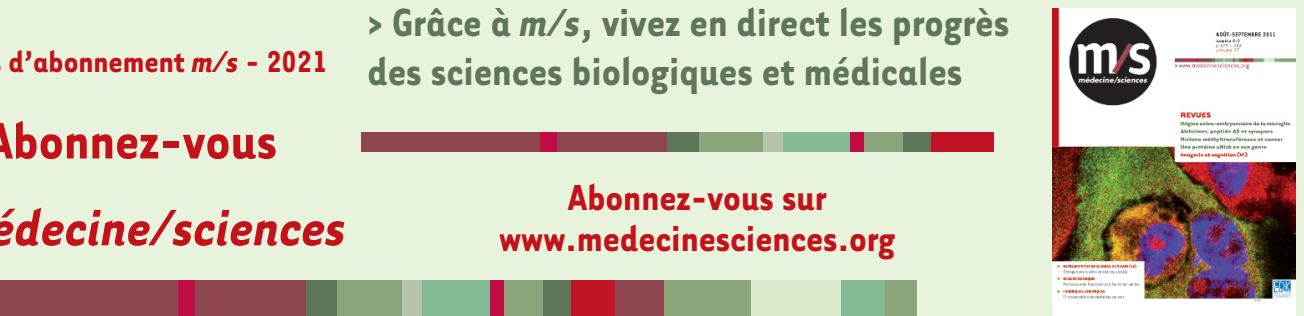